\title{
Barrier Option Pricing by Branching Processes
}

\author{
Georgi K. Mitov* \\ Svetlozar T. Rachev ${ }^{\dagger}$ \\ Young Shin Kim \\ Frank J. Fabozzi
}

\section{Georgi K. Mitov}

Institute of Mathematics and Informatics, Bulgarian Academy of Science

"Acad. G. Bonchev" Str., Bl. 8, 1113, Sofia, Bulgaria

and Senior Quant, FinAnalytica Inc.

E-mail: georgi.mitov@finanalytica.com

\section{Svetlozar T. Rachev}

Chair-Professor, Chair of Statistics, Econometrics and Mathematical Finance, School of Economics and Business Engineering, University of Karlsruhe and KIT, Kollegium am Schloss, Bau II, 20.12, R210, Postfach 6980, D-76128, Karlsruhe, Germany and Department of Statistics and Applied Probability, University of California, Santa Barbara,

and Chief-Scientist, FinAnalytica Inc.

E-mail: rachev@statistik.uni-karlsruhe.de

\section{Young Shin Kim}

Department of Statistics, Econometrics and Mathematical Finance, School of Economics and Business Engineering, University of Karlsruhe and KIT

Kollegium am Schloss, Bau II, 20.12, R210, Postfach 6980, D-76128, Karlsruhe, Germany E-mail: aaron.kim@statistik.uni-karlsruhe.de

\section{Frank J. Fabozzi}

Professor in the Practice of Finance,

Yale School of Management, 135 Prospect Street, Box 208200, New Haven, CT 06520-8200, USA

E-mail: frank.fabozzi@yale.edu

*Georgi Mitov is supported by the German Academic Exchange Service (DAAD) and in part by NSF- Bulgaria (grant VU-MI-105/20-05).

†Svetlozar Rachev gratefully acknowledges research support by grants from Division of Mathematical, Life and Physical Sciences, College of Letters and Science, University of California, Santa Barbara, the Deutschen Forschungsgemeinschaft, and the Deutscher Akademischer Austausch Dienst. 


\title{
Barrier Option Pricing by Branching Processes
}

\begin{abstract}
This paper examines the pricing of barrier options when the price of the underlying asset is modeled by branching process in random environment (BPRE). We derive an analytical formula for the price of an up-and-out call option, one form of a barrier option. Calibration of the model parameters is performed using market prices of standard call options. Our results show that the prices of barrier options that are priced with the BPRE model deviate significantly from those modeled assuming a lognormal process, despite the fact that for standard options, the corresponding differences between the two models are relatively small.
\end{abstract}

Key words: Barrier option, up-and-out call option, Bienayme-Galton-Watson branching process, branching process in random environment 


\section{Introduction}

Barrier options, also known as knock-in options or knock-out options, have become increasingly popular since they were first traded in the over-the-counter (OTC) market in 1967. Their popularity resulted in the introduction of barrier options on exchanges by 1991, first by the Chicago Board Option Exchange and then by the American Stock Exchange. The appealing feature of this exotic option product is twofold. First, it provides an investor with flexibility in a number of hedging strategies. Second, it is less expensive than standard options.

Barrier options are a type of path-dependent option which include down-and-out options, down-and-in options, up-and-out options, and up-and-in options. In 1973, Merton [20] provided a closed-form solution for down-and-out call options. Since then, closedform solutions for many European barrier options have been developed by Reiner and Rubinstein [23], Kunitomo and Ikeda [15], Carr [8], and Geman and Yor [14]. Numerical methods have been proposed to handle American or other more complex barrier options by Boyle and Lau [4], Ritchken [25], Broadie, Glasserman, and Kou [7], Boyle and Tian [6], and Zvan, Vetzal and Forsyth [31]. Most of the published papers on barrier options assume the underlying asset follows geometric Brownian motion. This implies that the price of the underlying asset is log-normally distributed as in the Black-Scholes [3] model. This assumption has several drawbacks.

The first drawback is that stock prices on the New York Stock Exchange (NYSE) were quoted in units of $\$ 1 / 8$, then in $\$ 1 / 16$, and now $\$ 0.01$. This discreteness of the stock price contradicts the continuous distribution assumption in the Black-Scholes formula. Second, it is evident that stock prices sometimes exhibit large jumps when some important news is disclosed. Third, extensive empirical evidence, pioneered by Mandelbrot [18, 19] and Fama [13], empirically documented that the logarithm of stock returns tend to be leptokurtic; that is, their distributions have thicker tails than the normal distribution derived from the geometric Brownian motion law. Furthermore, Black [2] noted the so-called "leverage effect," meaning that the volatility of stock returns tends to be negatively correlated with the price.

There are different ways to avoid these drawbacks of the lognormal model. One of 
them is to model the price of the underlying asset using different stochastic processes which possess some of the stylized facts reported for stock prices. Thus, Zhou [30] examines the case where the stock price follows a jump diffusion process. Valuation of barrier options in a constant elasticity of variance (CEV) model is treated in Boyle and Tian [5] and Davydov and Linetsky [10]. Shoutens and Symens [27] utilize Levy stochastic volatility models to price barrier options. Riberio and Webber [24] present a Monte Carlo algorithm for pricing barrier options with the variance gamma (VG) model.

In the present paper, we use a branching process in a random environment (BPRE) to model the stock price. This model of stock price movement was introduced by Epps [11] in 1996. The model, constructed by Bienayme-Galton-Watson branching process subordinated with a Poisson process, captures the stylized facts about stock return distributions. Specifically, the return distribution exhibits thick tails and a variance that decreases with the level of the stock price. The process also allows for possible jumps in stock prices, and takes into account the possibility of bankruptcy, something that is neglected in many other models.

Williams [29] provides an exact formula for the price of a European put option based on the BPRE model. Liu [17] applies the BPRE model to options on a sample of individual U.S. equities. Inferring the parameters from transaction prices of traded options, Liu finds that for the in-sample prediction, the model typically eliminates the smile effect that has been observed for option prices. The results are mixed when out-of-sample predictions are compared with the ad hoc version of Black-Scholes with moneyness-specific implicit volatilities. Liu does find evidence that the predictions are better for options on low-priced stocks, where the discreteness arising from the minimum tick size would seem to be more relevant.

In this paper, we derive a formula for the price of an up-and-out call option based on BPRE and compare the results with the prices based on the lognormal model. The paper is organized as follows. In Section 2, we review the mathematical definition of the BPRE model and its main properties and advantages. Since we rely on a methodology for the pricing of a European call option formulated by Mitov and Mitov [21] for the calibration of the equivalent martingale measure (EMM) parameters, we provide the 
formula at the end of Section 2. The formula for the price of an up-and-out call option is derived in Section 3. In Section 4, we report numerical results for barrier options on several stocks and the S\&P 500 index. We calibrate both BPRE and lognormal models to market prices of standard options, and subsequently price up-and-out call options. We then compare those prices based on the BPRE model and lognormal model. Section 5 summarizes our findings.

\section{The Model}

\subsection{Branching Processes}

Let us consider a Bienayme-Galton-Watson branching process, $Z_{n}, n=0,1,2, \ldots$ with a non-random number of ancestors $Z_{0}>0$, and the offspring probability distribution

$$
\begin{aligned}
& \mathbb{P}\left(Z_{n+1}=0 \mid Z_{n}=1\right)=(1-a), \\
& \mathbb{P}\left(Z_{n+1}=k \mid Z_{n}=1\right)=a p(1-p)^{k-1}, k=1,2, \ldots, \\
& 0<a<1, \quad 0<p<1 .
\end{aligned}
$$

The probability generating function (p.g.f.) $f(s)=\mathbb{E}\left[s^{Z_{1}} \mid Z_{0}=1\right]$ of the offspring distribution can be easily represented in terms of the distribution's factorial moments by

$$
f(s)=1-\frac{m(1-s)}{1+\frac{b}{2 m}(1-s)}, \quad s \in[0,1]
$$

where $m=a / p$ is the offspring mean and

$$
b=2 \frac{1-p}{p} m, \quad \sigma^{2}=b+m-m^{2}=\frac{a}{p}\left(\frac{(1-p)+(1-a)}{p}\right)
$$

are the offspring second moment and the offspring variance.

Such a function has fractional linear form. It is well known (see e.g. Sevastyanov [26]) that for the p.g.f. $f_{n}(s)=\mathbb{E}\left[s^{Z_{n}} \mid Z_{0}=1\right]$, the following relation holds

$$
f_{n}(s)=1-\frac{m^{n}(1-s)}{1+\frac{b}{2 m} \frac{1-m^{n}}{1-m}(1-s)} .
$$

In Section 3, we will need the probabilities

$$
\mathbb{P}\left(Z_{n}=k \mid Z_{0}=1\right), \quad k=0,1,2, \ldots, \quad n=1,2, \ldots
$$


Differentiating (1), we obtain

$$
f_{n}^{\prime}(s)=\frac{m^{n}}{\left[1+\frac{b\left(1-m^{n}\right)}{2 m(1-m)}(1-s)\right]^{2}},
$$

and for $k=2,3, \ldots$,

$$
f_{n}^{(k)}(s)=\frac{k ! m^{n}\left[\frac{b\left(1-m^{n}\right)}{2 m(1-m)}\right]^{k-1}}{\left[1+\frac{b\left(1-m^{n}\right)}{2 m(1-m)}(1-s)\right]^{k+1}} .
$$

From the properties of the p.g.f., it follows that

$$
\mathbb{P}\left(Z_{n}=k \mid Z_{0}=1\right)=\frac{f_{n}^{(k)}(0)}{k !}
$$

therefore we get for $k=1,2, \ldots$

$$
\mathbb{P}\left(Z_{n}=k \mid Z_{0}=1\right)=\frac{m^{n}\left[\frac{b\left(1-m^{n}\right)}{2 m(1-m)}\right]^{k-1}}{\left[1+\frac{b\left(1-m^{n}\right)}{2 m(1-m)}\right]^{k+1}} .
$$

Then the probability for extinction in the $n$-th generation is

$$
\begin{aligned}
\mathbb{P}\left(Z_{n}=0 \mid Z_{0}=1\right) & =1-\sum_{k=1}^{\infty} \frac{m^{n}\left[\frac{b\left(1-m^{n}\right)}{2 m(1-m)}\right]^{k-1}}{\left[1+\frac{b\left(1-m^{n}\right)}{2 m(1-m)}\right]^{k+1}} \\
& =1-\frac{m^{n}}{1+\frac{b\left(1-m^{n}\right)}{2 m(1-m)}}
\end{aligned}
$$

Substituting $s=1$ in (2), we get the first moment of the process

$$
\mathbb{E}\left[Z_{n} \mid Z_{0}=1\right]= \begin{cases}m^{n}, & m \neq 1 \\ 1, & m=1\end{cases}
$$

Substituting $k=2$ and $s=1$ in (3), we obtain the second factorial moment

$$
\mathbb{E}\left[Z_{n}\left(Z_{n}-1\right) \mid Z_{0}=1\right]= \begin{cases}b m^{n-1} \frac{1-m^{n}}{1-m}, & m \neq 1, \\ b n, & m=1 .\end{cases}
$$

If the process starts with $Z_{0}=I>1$ number of ancestors, it is a sum of $I$ independent and identically distributed branching processes, each of them beginning with an ancestor. Therefore,

$$
\mathbb{E}\left[s^{Z_{n}} \mid Z_{0}=I\right]=\left(f_{n}(s)\right)^{I}
$$


and we can calculate for $k=1,2, \ldots$

$$
\mathbb{P}\left(Z_{n}=k \mid Z_{0}=I\right)=\left.\frac{d^{k}\left(f_{n}(s)\right)^{I}}{d s^{k}}\right|_{s=0} .
$$

Instead of using the derivatives, we suggest the following simple iterative procedure. For any $n>0, Z_{0}=I>1$, and $K \geq 0$, calculate:

(i) $\mathbb{P}\left(Z_{n}=k \mid Z_{0}=1\right)$ for $k=0,1,2, \ldots, K$ by (4) and (5).

(ii) For $Z_{0}=2$, the process is the sum of two independent processes each of which begins with $Z_{0}=1$ particle. Therefore,

$$
\mathbb{P}\left(Z_{n}=k \mid Z_{0}=2\right)=\sum_{j=0}^{k} \mathbb{P}\left(Z_{n}=j \mid Z_{0}=1\right) \mathbb{P}\left(Z_{n}=k-j \mid Z_{0}=1\right),
$$

for all $k=0,1,2, \ldots, K$.

(iii) For $Z_{0}=I, \quad I \geq 3$, the process is the sum of two independent processes one of which starts with $Z_{0}=I-1$ and the other starts with $Z_{0}=1$ particle. That is,

$$
\mathbb{P}\left(Z_{n}=k \mid Z_{0}=I\right)=\sum_{j=0}^{k} \mathbb{P}\left(Z_{n}=j \mid Z_{0}=I-1\right) \mathbb{P}\left(Z_{n}=k-j \mid Z_{0}=1\right) .
$$

for all $k=0,1,2, \ldots, K$.

Note that the calculations can be easily performed using numerical software if for a fixed $n$ and $K$, the probabilities $p_{1 k}(n)=\mathbb{P}\left(Z_{n}=k \mid Z_{0}=1\right), k=0,1,2, \ldots, K$, calculated in the point (i) are entered in an upper triangular matrix as follows

$$
\mathbf{P}(n)=\left(\begin{array}{ccccc}
p_{10}(n) & p_{11}(n) & p_{12}(n) & \ldots & p_{1 K}(n) \\
0 & p_{10}(n) & p_{11}(n) & \ldots & p_{1, K-1}(n) \\
0 & 0 & p_{10}(n) & \ldots & p_{1, K-2}(n) . \\
\ldots & \ldots & \ldots & \ldots & \ldots \\
0 & 0 & 0 & \ldots & p_{10}(n)
\end{array}\right)
$$

It is not difficult to see that the probabilities $p_{I k}(n)=\mathbb{P}\left(Z_{n}=k \mid Z_{0}=I\right), k=$ $0,1,2 \ldots, K$ are equal to the $(1, k)$-th element of the $I$-th power of matrix $\mathbf{P}(n)$.

\subsection{Branching process in random environment as a price pro- cess}

Assume now that on the common probability space $(\Omega, \mathcal{A}, \mathbb{P})$ are given (i) a supercritical $(m>1)$ Bienayme-Galton-Watson branching process $Z_{n}, n=0,1,2, \ldots$ defined in the previous section and (ii) an independent of it Poisson process $N(t), t \geq 0$ with 
intensity $\lambda>0$. Following Epps [11] or [12], define the randomly indexed branching process, which is in fact a branching process in random environment (BPRE),

$$
S(t)=Z_{N(t)}, t \geq 0 \text {. }
$$

Here $S(t)$ represents the price of one share of stock at time $t$ measured in units of minimum price movements (for example \$0.01). Equity prices are then viewed as consisting of an integer number of "price particles." In each period, each "price particle" of equity price produces a random number of offspring "price particles," the aggregate number of which comprises the equity price in the next period. Hence, by allowing a random number of generations to occur in each period, the BPRE model generates prices in continuous time.

The independence of $Z_{n}$ and $N(t)$ yields the following formula for the p.g.f. of the process $S(t)=Z_{N(t)}$, starting with $Z_{0} \equiv S(0) \geq 1$ ancestors

$$
\begin{gathered}
\Phi(t, s)=\sum_{n=0}^{\infty} \frac{(\lambda t)^{n}}{n !} e^{-\lambda t}\left(f_{n}(s)\right)^{S(0)} \\
=\sum_{n=0}^{\infty} \frac{(\lambda t)^{n}}{n !} e^{-\lambda t}\left(1-\frac{m^{n}(1-s)}{1+\frac{b}{2 m} \frac{1-m^{n}}{1-m}(1-s)}\right)^{S(0)} .
\end{gathered}
$$

Using the p.g.f. and formulas (6) and (7), after some calculations we derive the following formulas for the mean and the variance of the process $S(t)$

$$
\begin{gathered}
M(t)=\mathbb{E}[S(t) \mid S(0)]= \begin{cases}S(0) e^{\lambda t(m-1)}, & m \neq 1, \\
S(0), & m=1,\end{cases} \\
\sigma^{2}(t)=\operatorname{Var}[S(t) \mid S(0)]= \begin{cases}S(0)^{2}\left[e^{\lambda t\left(m^{2}-1\right)}-e^{2 \lambda t(m-1)}\right] \\
+\frac{S(0) \sigma^{2}\left[e^{\lambda t\left(m^{2}-1\right)}-e^{\lambda t(m-1)}\right]}{m(m-1)}, & m \neq 1 \\
S(0) \sigma^{2} \lambda t, & m=1 .\end{cases}
\end{gathered}
$$

These formulas allow us to examine in detail some of the main properties of the total return $R(t)=[S(t)-S(0)] / S(0)$ over a period $(0, t)$. The first two moments of the return distribution have the following form:

$$
\mathbb{E}[R(t) \mid S(0)]= \begin{cases}e^{\lambda t(m-1)}-1, & m \neq 1 \\ 0, & m=1\end{cases}
$$




$$
\operatorname{Var}[R(t) \mid S(0)]= \begin{cases}e^{\lambda t\left(m^{2}-1\right)}-e^{2 \lambda t(m-1)} & \\ +\frac{1}{S(0)}\left(\frac{\sigma^{2}}{m(m-1)}\left(e^{\lambda t\left(m^{2}-1\right)}-e^{\lambda t(m-1)}\right)\right), & m \neq 1 \\ \frac{b \lambda t}{S(0)}, & m=1 .\end{cases}
$$

The coefficient of $1 / S(0)$ in the variance representation is positive, because we examine a supercritical Bienayme-Galton-Watson branching process, that is $m>1$. Therefore, the variance of the return is inversely related to the stock price. As a result, the so-called "leverage effect" is built into the model.

Formulas for the skewness $\gamma_{1}[R(t)]$ and kurtosis $\gamma_{2}[R(t)]$ are more complicated, but if $m=1$ the standardized third and forth moments have the simple forms:

$$
\begin{gathered}
\gamma_{1}[R(t)]=\frac{\mathbb{E}\left[R(t)^{3} \mid S(0)\right]}{(\operatorname{Var}[R(t) \mid S(0)])^{3 / 2}}=\frac{3 \sigma}{2 \sqrt{S(0)}}\left(\sqrt{\lambda t}+\frac{1}{\sqrt{\lambda t}}\right) \\
\gamma_{2}[R(t)]=\frac{\mathbb{E}\left[R(t)^{4} \mid S(0)\right]}{\left(\mathbb{E}\left[R(t)^{2} \mid S(0)\right]\right)^{2}}-3=\frac{3}{\lambda t}+\frac{1}{S(0) \lambda t}+\frac{2 \sigma^{2}}{S(0)}\left(\lambda t+1+\frac{1}{\lambda t}\right) .
\end{gathered}
$$

The estimates of $m$ with daily frequency stock data are greater than but very close to one, so that the last two expressions give a close approximation to the higher moments over short trading periods. Skewness is always positive, decreasing in $S(0)$ and generally increasing with the period of the return $t$. Kurtosis is always positive, which means that it has fatter tails relative to the normal distribution, and it is a decreasing function of $t$ for $t$ less than $\sqrt{3 S(0)+2 \sigma^{2}+1} /(\lambda \sigma \sqrt{2})$. The last expression is in general greater than 22, which means that the daily returns have fatter tails than the weekly and monthly returns. This characteristic has been observed for equity prices and is called aggregational normality (see Cont and Tankov [9]).

\subsection{Option Pricing by BPRE}

The discreteness of $S(t)$ under BPRE dynamics makes it impossible to replicate nonlinear payoff structures with only the underlying asset and riskless bonds. Accordingly, there is not a unique EMM within which derivatives can be priced as discounted expected values. Recall that the process $Z_{n} m^{-n}, n=0,1,2, \ldots$ is a martingale (see, for example, 
Athreya and Ney [1]). The process $S(t)$ has a similar property, enabling us to find the EMM required for the option pricing.

Theorem 1. Under conditions (i) and (ii) assumed in Section 2.2, the process $S(t) e^{-\lambda t(m-1)}$, $t \geq 0$ is a nonnegative martingale.

The proof is as following. Since $S(t), t \geq 0$ is a continuous-time Markov chain, we have that for every $n$ and every sequence $0 \leq t_{1}<t_{2}<\ldots<t_{n}<t$

$$
\mathbb{E}\left[S(t) \mid S\left(t_{n}\right), \ldots, S\left(t_{2}\right), S\left(t_{1}\right)\right]=\mathbb{E}\left[S(t) \mid S\left(t_{n}\right)\right]
$$

Therefore, it is sufficient to prove that for $t \geq 0$ and $\tau \geq 0$

$$
\mathbb{E}\left[e^{-\lambda(t+\tau)(m-1)} S(t+\tau) \mid e^{-\lambda t(m-1)} S(t)\right]=e^{-\lambda t(m-1)} S(t) .
$$

Let us note first that

$$
\mathbb{E}\left[e^{-\lambda(t+\tau)(m-1)} S(t+\tau) \mid e^{-\lambda t(m-1)} S(t)\right]=e^{-\lambda(t+\tau)(m-1)} \mathbb{E}[S(t+\tau) \mid S(t)]
$$

Using the fact that the processes $N(t)$ and $Z_{n}$ are time-homogeneous, and using the main property of branching processes, we have

$$
\begin{aligned}
& \mathbb{E}[S(t+\tau) \mid S(t)]=\mathbb{E}\left[Z_{N(t+\tau)} \mid Z_{N(t)}\right] \\
= & \mathbb{E}\left[\sum_{i=1}^{Z_{N(t)}} Z_{N(t+\tau)-N(t)}^{i} \mid Z_{N(t)}\right] \\
= & \mathbb{E}\left[\sum_{i=1}^{Z_{N(t)}} Z_{N(\tau)}^{i} \mid Z_{N(t)}\right]=Z_{N(t)} \mathbb{E}\left[Z_{N(\tau)}^{1}\right]=S(t) \mathbb{E}\left[Z_{N(\tau)}^{1}\right],
\end{aligned}
$$

where $Z_{N(\tau)}^{i}$ are independent and identically distributed branching processes that are independent of $Z_{N(t)}$, and each of them starting with one particle. Using (8), with $S(0)=1$, we get

$$
\begin{aligned}
& \mathbb{E}[S(t+\tau) \mid S(t)]=S(t) \mathbb{E}\left[Z_{N(\tau)} \mid Z_{0}=1\right] \\
= & S(t) \mathbb{E}[S(\tau) \mid S(0)=1]=S(t) e^{\lambda \tau(m-1)} .
\end{aligned}
$$

Combining (9), (10), and (11) we complete the proof.

From (8) it follows that the discounted stock price process $S(t) e^{-r t}, t \in[0, T]$ has mean

$$
\mathbb{E}\left[S(t) e^{-r t} \mid S(0)\right]=e^{[\lambda(m-1)-r] t} S(0) .
$$


Using Theorem 1, we can state that discounted stock price process $S(t) e^{-r t}$ will be a martingale if the parameters of the distribution of $S(t)$ are such that

$$
\lambda(m-1)=r \Leftrightarrow \lambda \frac{a-p}{p}=r \quad \Leftrightarrow \quad a=p(1+r / \lambda) .
$$

Utilizing the last relation we define EMM $\mathbb{Q}$ as follows:

1. We define $\mathbb{Q}$ to be identically equal to the real measure $\mathbb{P}$ on the elementary sets of the Poisson process, i.e. on sets of the form $\left\{N_{t_{0}}=n_{0}, N_{t_{1}}=n_{1}, \ldots, N_{t_{k}}=n_{k}\right\}$.

2. We define $\mathbb{Q}$ on the elementary sets of the branching process by

$$
\begin{aligned}
& \mathbb{Q}\left(Z_{n+1}=0 \mid Z_{n}=1\right)=(1-\hat{a}), \\
& \mathbb{Q}\left(Z_{n+1}=k \mid Z_{n}=1\right)=\hat{a} p(1-p)^{k-1}, k=1,2, \ldots, \\
& 0<\hat{a}<1, \quad 0<p<1,
\end{aligned}
$$

where $\hat{a}$ satisfies equation (12). In the case when $p(1+r / \lambda) \geq 1$, we also have to change the parameter $p, 0<p<1$ in order to keep $\hat{a} \in(0,1)$.

The choice of $\hat{a}$ guarantees that $0<\hat{a}<1$ and, therefore, we do not change the zero measure sets; that is, all sets that have zero measure with respect to the real measure $\mathbb{P}$ have zero measure with respect to $\mathbb{Q}$. Consequently, these two measures are equivalent. From the definition of $\mathbb{Q}$, it is easily seen that the discounted process $S(t) e^{-r t}$ is a martingale under $\mathbb{Q}$. Henceforth, we will work exclusively with the riskneutral probability and will denote it simply by $\mathbb{P}$. In the numerical examples presented in the Section 4 we keep the estimated values of $p$ and $\lambda$ and choose the value of the parameter $a$ so that equation (12) is satisfied.

Following Mitov and Mitov [21], we derive the formula for the price of a European call option

$$
\begin{aligned}
& C(0)=e^{-r T} \mathbb{E}[\max \{S(T)-K, 0\} \mid S(0)] \\
& =\quad e^{-r T}\left[\sum_{k=K+1}^{\infty} k \mathbb{P}(S(T)=k \mid S(0))-K \mathbb{P}(S(T)>K \mid S(0))\right] \\
& =\quad e^{-r T}\left[\mathbb{E}[S(T) \mid S(0)]-\sum_{k=1}^{K} k \mathbb{P}(S(T)=k \mid S(0))-K(1-\mathbb{P}(S(T) \leq K \mid S(0)))\right] \\
& =\quad e^{-r T}[\mathbb{E}[S(T) \mid S(0)]-K]+e^{-r T} \sum_{k=1}^{K}(K-k) \mathbb{P}(S(T)=k \mid S(0)),
\end{aligned}
$$


where the expectations are taken with respect to the risk-neutral measure $\mathbb{P}$ (the riskneutral probability). Using the relation

$$
\mathbb{P}(S(T)=k \mid S(0))=\sum_{n=0}^{\infty} \frac{(\lambda T)^{n}}{n !} e^{-\lambda T} \mathbb{P}\left(Z_{n}=k \mid Z_{0}=S(0)\right), k=0,1,2, \ldots,
$$

the fact that $S(t) e^{-\lambda t(m-1)}$ is a nonnegative martingale and (12), we obtain the following exact formula for the price of a European call option

$$
\begin{gathered}
C(0)=S(0)-e^{-r T} K \\
+e^{-(r+\lambda) T} \sum_{n=0}^{\infty} \frac{(\lambda T)^{n}}{n !} \sum_{k=0}^{K}(K-k) \mathbb{P}\left(Z_{n}=k \mid Z_{0}=S(0)\right),
\end{gathered}
$$

where $K$ is the strike price, $T$ is time to maturity of the option, $r$ is risk-free interest rate, $S(0)$ is the current stock price, and $\lambda$ is the intensity of the Poisson process.

For practical purposes Mitov and Mitov use the approximation

$$
\begin{aligned}
& C(0) \approx S(0)-K e^{-r T} \\
+ & e^{-(r+\lambda) T} \sum_{n=0}^{N} \frac{(\lambda T)^{n}}{n !} \sum_{k=0}^{K}(K-k) \mathbb{P}\left(Z_{n}=k \mid Z_{0}=S(0)\right),
\end{aligned}
$$

where number $N$ can be determined in such a way that the error from the approximation will be less than $\varepsilon$. The probabilities $\mathbb{P}\left(Z_{n}=k \mid Z_{0}=S(0)\right)$ for $k=0,1,2, \ldots K$ and $n=1,2, \ldots N$, are calculated by the iterative procedure described at the end of Section 2.1 .

\section{$3 \quad$ Barrier Option Pricing}

There are several types of barrier options. Some "knock out" (i.e., they become worthless) when the underlying asset price crosses a barrier. If the underlying asset price begins below the barrier and must cross above it to cause the knock-out, the option is said to be up-and-out. A down-and-out option has the barrier below the initial asset price and knocks out if the asset price falls below the barrier. Other options "knock in" at a barrier (i.e., there is no payoff unless asset price crosses a barrier). Knock-in options also fall into two classes, up-and-in and down-and-in. The payoff at expiration for barrier options is typically either that of a put or a call. There exist more complex barrier options, but in this section, we focus only on an up-and-out call option on a BPRE 
process. The methodology we develop can be also applied to up-and-in call options. For the rest we can use in-out parity ${ }^{1}$ and the price of the standard call option given in (13) and (14).

The price at maturity date of an up-and-out European call option with strike price $K$ and barrier level $B$ is:

$$
C^{u o}(T) \stackrel{\text { def }}{=}\left\{\begin{array}{l}
\max (0, S(T-K)), \quad M(T) \leq B \\
0, \quad M(T)>B
\end{array}\right.
$$

where $M(T)=\max _{0 \leq t \leq T} S(t)$. Applying martingale pricing, the price of the option at the current moment is

$$
C^{u o}(0)=e^{-r T} \mathbb{E}\left[C^{u o}(T) \mid S(0)\right],
$$

Recall that the expectation is taken with respect to the risk-neutral measure $\mathbb{P}$. Therefore

$$
\begin{gathered}
C^{u o}(0)=e^{-r T} \mathbb{E}\left[\max (0, S(T)-K) I_{\{M(T) \leq B\}}\right] \\
=e^{-r T} \sum_{j=0}^{B} \max (0, j-K) \mathbb{P}(S(T)=j, M(T) \leq B \mid S(0)) .
\end{gathered}
$$

Taking into account that $B>K$, we obtain ${ }^{2}$

$$
C^{u o}(0)=e^{-r T} \sum_{j=K}^{B}(j-K) \mathbb{P}(S(T)=j, M(T) \leq B \mid S(0)) .
$$

Using the relation

$$
\mathbb{P}(S(T)=k, M(T) \leq B \mid S(0))=\sum_{n=0}^{\infty} \frac{(\lambda T)^{n}}{n !} e^{-\lambda T} \mathbb{P}\left(Z_{n}=k, M_{n} \leq B \mid Z_{0}=S(0)\right),
$$

for $k=0,1,2, \ldots$, we can write

$$
\begin{gathered}
C^{u o}(0)=e^{-(r+\lambda) T} \sum_{n=0}^{\infty} \frac{(\lambda T)^{n}}{n !} \sum_{j=K}^{B}(j-K) \mathbb{P}\left(Z_{n}=j, M_{n} \leq B \mid Z_{0}=S(0)\right) \\
=e^{-(r+\lambda) T} \sum_{n=0}^{N} \frac{(\lambda T)^{n}}{n !} \sum_{j=K}^{B}(j-K) \mathbb{P}\left(Z_{n}=j, M_{n} \leq B \mid Z_{0}=S(0)\right)
\end{gathered}
$$

\footnotetext{
${ }^{1}$ If we combine one "in" option and one "out" barrier option with the same strikes and expirations, we get the price of a standard option. This is the barrier option's equivalence to the put-call relationship for standard options.

${ }^{2}$ If barrier level $B$ is less than or equal to the strike price $K$, the price of an up-and-out call option is zero. Therefore, we examine only the case where $B>K$.
} 


$$
+e^{-(r+\lambda) T} \sum_{n=N+1}^{\infty} \frac{(\lambda T)^{n}}{n !} \sum_{j=K}^{B}(j-K) \mathbb{P}\left(Z_{n}=j, M_{n} \leq B \mid Z_{0}=S(0)\right) .
$$

But since

$$
\sum_{j=K}^{B}(j-K) \mathbb{P}\left(Z_{n}=j, M_{n} \leq B \mid Z_{0}\right) \leq \sum_{j=K}^{B}(j-K) 1=\frac{(B-K)(B-K+1)}{2}
$$

then

$$
\begin{gathered}
e^{-(r+\lambda) T} \sum_{n=N+1}^{\infty} \frac{(\lambda T)^{n}}{n !} \sum_{j=K}^{B}(j-K) \mathbb{P}\left(Z_{n}=j, M_{n} \leq B \mid Z_{0}=S(0)\right) \\
\leq e^{-(r+\lambda) T} \sum_{n=N+1}^{\infty} \frac{(\lambda T)^{n}}{n !} \frac{(B-K)(B-K+1)}{2}
\end{gathered}
$$

Infinite series can be calculated with an arbitrary precision $\varepsilon>0$, hence the number $N$ can be determined in such a way that

$$
e^{-(r+\lambda) T} \frac{(B-K)(B-K+1)}{2} \sum_{n=N+1}^{\infty} \frac{(\lambda T)^{n}}{n !}<\varepsilon,
$$

provided the values of $r, \lambda, T, B, K$, and $S(0)$ are known. Therefore, we can use the approximation

$$
C^{u o}(0) \approx e^{-(r+\lambda) T} \sum_{n=0}^{N} \frac{(\lambda T)^{n}}{n !} \sum_{j=K}^{B}(j-K) \mathbb{P}\left(Z_{n}=j, M_{n} \leq B \mid Z_{0}=S(0)\right),
$$

with an error less than $\varepsilon$.

The method for the calculation of the probabilities $\mathbb{P}\left(Z_{n}=j, M_{n} \leq B \mid Z_{0}=S(0)\right)$ used in equation (15), is given in the following theorem.

Theorem 2. If $\left\{Z_{n}, n=0,1,2, \ldots\right\}$ is a Bienayme-Galton-Watson branching process with $p_{i j}=\mathbb{P}\left(Z_{1}=j \mid Z_{0}=i\right), i, j=0,1,2, \ldots$ and $B$ is a positive integer then the probability

$$
\mathbb{P}\left(Z_{n}=j, M_{n} \leq B \mid Z_{0}=i\right)
$$

is given by the $(i, j)$-th element of the $n$-th power of matrix $\mathbf{P}(B, 1)$, where

$$
p_{i j}(B, 1)=\mathbb{P}\left(Z_{1}=j, M_{1} \leq B \mid Z_{0}=i\right)=\left\{\begin{array}{l}
p_{i j}, \quad 1 \leq i, j \leq B \\
1, \quad i, j=0, \\
0, \quad i=0, j>0 \\
0, \quad B<i, j
\end{array}\right.
$$


The proof of this theorem is as follows. For the conditional probabilities $\mathbb{P}\left(Z_{2}=\right.$ $\left.j, M_{2} \leq B \mid Z_{0}=i\right)$, calculated for the second generation, we can write:

$$
\begin{aligned}
& p_{i j}(B, 2)= \mathbb{P}\left(Z_{2}=j, M_{2} \leq B \mid Z_{0}=i\right) \\
&= \sum_{l=0}^{\infty} \mathbb{P}\left(Z_{2}=j, M_{2} \leq B \mid Z_{1}=l\right) \mathbb{P}\left(Z_{1}=l, M_{1} \leq B \mid Z_{0}=i\right) \\
&= \sum_{l=1}^{B} \mathbb{P}\left(Z_{2}=j, M_{2} \leq B \mid Z_{1}=l\right) \mathbb{P}\left(Z_{1}=l, M_{1} \leq B \mid Z_{0}=i\right) \\
&= \sum_{l=1}^{B} \mathbb{P}\left(Z_{1}=j, M_{1} \leq B \mid Z_{0}=l\right) \mathbb{P}\left(Z_{1}=l, M_{1} \leq B \mid Z_{0}=i\right) \\
&=\sum_{l=1}^{B} p_{i l} p_{l j}, \quad 1 \leq i, j \leq B .
\end{aligned}
$$

Therefore $\mathbf{P}(B, 2)=\left(p_{i j}(B, 2)\right)_{i, j=1, \ldots, B}=\mathbf{P}(B, 1)^{2}$. Repeating the above procedure, we can deduce by induction that $\mathbf{P}(B, n)=\mathbf{P}(B, 1)^{n}$, which proves the statement of the theorem.

The one-step transition probabilities $p_{i j}=\mathbb{P}\left(Z_{1}=j \mid Z_{0}=i\right), \quad i, j=0,1,2, \ldots$, are calculated using the iterative procedure described at the end of Section 2.1.

\section{Numerical Examples}

In this section, we compare the prices of up-and-out barrier options calculated under the lognormal and BPRE models. According to Musiela and Rutkowski [22], any mathematical model used for pricing exotic options should be marked-to-market; that is, at any given date it should reproduce with the desired precision the current market prices of liquid options. Standard options are the most liquid options and therefore we mark-to-market our model by minimizing the sum-of-squares distance between the theoretical option values and market prices of standard options. The estimated parameters are then fed into the lognormal and BPRE models to calculate the up-and-out barrier option prices.

\subsection{Parameters Estimation}

We estimate the EMM parameters of the BPRE and lognormal model for the S\&P 500 Index (SPX) and the following three stocks: Intel (INTC), Microsoft (MSFT), and 
Amazon.com (AMZN). The data were obtained from Option Metrics's IvyDB in the Wharton Research Data Services. We selected five mid-month Wednesdays (June 8, 2005; July 13, 2005; August 10, 2005; September 14, 2005; October 12, 2005) and estimate the EMM parameters of the stock price processes. The market option prices are computed by using the Black-Scholes formula with the implied volatilities and dividends given by IvyDB. For the daily risk-free rate, we select the appropriate zero-coupon rate supplied by IvyDB and convert it to a continuous-compound rate.

For each model, we estimate the EMM parameters using the method of least-squares calibration with a prior (see Kim et al. [16]); that is, we estimate them by nonlinear least squares minimization under the EMM condition (12). The BPRE model has three EMM parameters to be estimated and one restriction in its EMM condition, while the lognormal model has one EMM parameter and there are no additional restrictions. Hence, the BPRE model has two free parameter $(\lambda$ and $p)$, while the lognormal model has only one free parameter $(\sigma)$ for the estimation.

To measure the performance of the prices estimated from the two models, we use average absolute error (AAE), average percentage error (APE), and root-mean square error (RMSE). These are defined as follows (Schoutens [28]):

$$
\begin{gathered}
A A E=\sum_{\text {options }} \frac{\mid \text { market price }- \text { model price } \mid}{\text { number of options }}, \\
A P E=\frac{1}{\text { mean option price } \sum_{\text {options }} \frac{\mid \text { market price }- \text { model price } \mid}{\text { number of options }},} \\
R M S E=\sqrt{\sum_{\text {options }} \frac{(\text { market price }- \text { model price })^{2}}{\text { number of options }}} .
\end{gathered}
$$

The values of these errors and estimated parameters for both models are presented in Tables 1 through 4 .

\subsection{Up-and-Out Call Option Prices}

Using the estimated parameters, we calculate the prices of up-and-out call options with different maturities and strikes, while the barrier level is the same for all cases. It 
is chosen to be greater than all of the strikes. The results for SPX, AMZN, INTC, and MSFT are reported in Tables 5 through 8. In the same tables, we report the results for standard call options with the same strikes and maturities. The BPRE prices of barrier and standard options are calculated by (15) and (14), respectively. The prices of up-and-out call options for the BPRE process deviate significantly from those for the lognormal process, while the prices of the corresponding standard options are similar. For example, if we examine the options on SPX with 72 days to maturity (Table 5), we can see that the maximum percentage differences in barrier option prices is $139.53 \%$, while for the standard options it is $1.15 \%$.

It can be seen that the BPRE prices are less than the lognormal prices for the up-andout options with shorter maturity (five days). This is due to the fact that BPRE returns have fatter tails for smaller periods and therefore we have greater probability of reaching the barrier level with respect to the lognormal model. In other words, if we have the same value for the standard call option, we will get the smaller value for an up-and-out barrier option using BPRE when the maturity period is shorter. It is interesting to note that the BPRE model produces greater prices for the options with 38 and 72 days to expiration, compared to the lognormal model. Figures 1 through 4 show the barrier option prices as a function of time to maturity. This could be partially explained by the presence of the so called "aggregational normality" effect, i.e. the distribution of the return gets closer to the normal distribution as the length of its period increases. Therefore, the difference in the tails of the distribution has a significant impact on barrier option prices.

\section{Conclusions}

In this paper, we present a simple and easy-to-use method for computing accurate estimates of up-and-out call option prices when the underlying stock process is modeled by BPRE. We demonstrate that the prices of barrier options for the BPRE process can deviate significantly from those calculated assuming a lognormal process, even if we have similar values for the corresponding standard options. We find that there is different behavior for the prices computed from the BPRE model with respect to the maturity period. For shorter maturities, the BPRE model gives smaller values compared 
to the lognormal model, while for the longer times to expiration we observe the opposite.

\section{References}

[1] K. Athreya and P. Ney, Branching Processes, Springer (1972).

[2] F. Black, Studies of stock market volatility changes, Proceedings of the American Statistical Association, Business and Economic Statistics Section (1976) 177-181.

[3] F. Black and M. Scholes, The pricing of options and corporate liabilities, Journal of Political Economy 81 (1973) 637-654.

[4] P. Boyle and I. Lau, Bumping up against the barrier with the binomial method, Journal of Derivatives 1 (1994) 6-14.

[5] P. Boyle and Y. Tian, Pricing lookback and barrier options under the CEV process, Journal of Financial and Quantitative Analysis 34 (1999) 241-264.

[6] P. Boyle and Y. Tian, An explicit finite difference approach to the pricing of barrier options, Applied Mathematical Finance 5 (1998) 17-43.

[7] M. Broadie, P. Glasserman, and S. Kou, A continuity correction for discrete barrier options, Mathematical Finance 7 (1997) 325-349.

[8] P. Carr, Two extensions to barrier option valuation, Applied Mathematical Finance 2 (1995) 173-209.

[9] R. Cont and P. Tankov, Financial Modelling with Jump Processes, Chapman and Hall / CRC, (2004).

[10] D. Davydov and V. Linetsky, Pricing and hedging path-dependent options under the CEV process, Management Science 47 (2001) 949-965.

[11] T.W. Epps, Stock prices as branching processes, Communications in Statistics: Stochastic Models 12 (1996) 529-558.

[12] T.W. Epps, Pricing Derivative Securities : Second Edition, World Scientific Publishing Company (2007). 
[13] E. Fama, Mandelbrot and the stable paretian hypothesis, Journal of Business 36 (1963) 420-429.

[14] H. Geman and M. Yor, Pricing and hedging double-barrier options: A probabilistic approach, Mathematical Finance 6 (1996) 365-378.

[15] N. Kunitomo and M. Ikeda, Pricing options with curved boundaries, Mathematical Finance 2 (1992) 275-298

[16] Y. S. Kim, S. T. Rachev, M. L. Bianchi, and F. J. Fabozzi, Financial market models with Levy processes and time-varying volatility, Journal of Banking and Finance, 32 (2008) 1363-1378

[17] W. Liu, Option Pricing with Pure Jump Models, Ph.D. Dissertation University of Virginia (2003).

[18] B. B. Mandelbrot, New methods in statistical economics, Journal of Political Economy, 71 (1963) 421-440.

[19] B. B. Mandelbrot, The variation of certain speculative prices, Journal of Business, 36 (1963) 394-419.

[20] R. C. Merton, Theory of rational option pricing, Bell Journal of Economics and Management Science, 4 (1973) 141-183.

[21] G.K. Mitov and K.V. Mitov, An option pricing formula based on branching processes, Pliska - Studia Mathematica Bulgarica 18 (2006) 213-224.

[22] M. Musiela and M. Rutkowski, Martingale Methods in Financial Modeling: Second Edition, Springer Verlag, (2005).

[23] E. Reiner and M. Rubinstein, Breaking down the barriers, Risk 4 (1991) 28-35.

[24] C. Ribeiro and N. Webber, Valuing path-dependent options in the variance-gamma model by Monte Carlo with a gamma bridge, Journal of Computational Finance 7 (2004) 81-100. 
[25] P. Ritchken, On pricing barrier options, Journal of Derivatives 3 (1994) 19-28.

[26] B.A. Sevastyanov, Branching Processes, Nauka, (1971).

[27] W. Schoutens and S. Symens, The pricing of exotic options by Monte-Carlo simulations in a Levy market with stochastic volatility. International Journal for Theoretical and Applied Finance 6 (2003) 839-864.

[28] W. Schoutens, Levy Processes in Finance: Pricing Financial Derivatives. John Wiley \& Sons. (2003).

[29] T. Williams, Option Pricing and Branching Processes, Ph.D. Dissertation University of Virginia (2001).

[30] C. Zhou, Path-dependent option valuation when the underlying path is discontinuous. Journal of Financial Engineering 8 (1999) 73-97.

[31] R. Zvan, K. R. Vetzal, P. A. Forsyth, PDE methods for pricing barrier options. Journal of Economic Dynamics and Control 24 (2000) 1563-1590. 
Table 1: SPX : Estimated EMM parameters and respective estimation errors

\begin{tabular}{|c|c|c|c|c|c|c|c|}
\hline \multirow{2}{*}{$\begin{array}{l}\text { Date } \\
08-J U N-2005\end{array}$} & \multirow{2}{*}{$\begin{array}{l}\text { Model } \\
\text { BPRE }\end{array}$} & \multicolumn{3}{|c|}{ Parameters } & \multirow[t]{2}{*}{$\mathrm{AAE}$} & \multirow[t]{2}{*}{$\mathrm{APE}$} & \multirow[t]{2}{*}{ RMSE } \\
\hline & & $\mathrm{p}$ & $\mathrm{a}$ & $\lambda$ & & & \\
\hline & & 0.9891 & 0.9892 & 1.3968 & 1.1457 & 0.0120 & 1.3836 \\
\hline & lognormal & $\sigma$ & & & & & \\
\hline & & 0.0827 & & & 1.0391 & 0.0109 & 1.3387 \\
\hline \multirow[t]{4}{*}{ 13-JUL-2005 } & BPRE & $\mathrm{p}$ & $\mathrm{a}$ & $\lambda$ & & & \\
\hline & & 0.9913 & 0.9914 & 1.3202 & 1.3723 & 0.0129 & 1.845 \\
\hline & lognormal & $\sigma$ & & & & & \\
\hline & & 0.2009 & & & 1.3375 & 0.0125 & 1.8392 \\
\hline \multirow[t]{4}{*}{ 10-AUG-2005 } & BPRE & $\mathrm{p}$ & $\mathrm{a}$ & $\lambda$ & & & \\
\hline & & 0.9885 & 0.9886 & 1.4149 & 0.7787 & 0.0085 & 0.9559 \\
\hline & lognormal & $\sigma$ & & & & & \\
\hline & & 0.0822 & & & 0.7947 & 0.0087 & 0.9638 \\
\hline \multirow[t]{4}{*}{ 14-SEP-2005 } & BPRE & $\mathrm{p}$ & $\mathrm{a}$ & $\lambda$ & & & \\
\hline & & 0.9883 & 0.9884 & 1.4197 & 1.1849 & 0.0108 & 1.4335 \\
\hline & lognormal & $\sigma$ & & & & & \\
\hline & & 0.0831 & & & 1.1807 & 0.0107 & 1.4324 \\
\hline \multirow[t]{4}{*}{ 12-OCT-2005 } & BPRE & $\mathrm{p}$ & $\mathrm{a}$ & $\lambda$ & & & \\
\hline & & 0.9887 & 0.9888 & 1.6182 & 0.6793 & 0.0473 & 0.9077 \\
\hline & lognormal & $\sigma$ & & & & & \\
\hline & & 0.0917 & & & 0.4178 & 0.0295 & 0.7989 \\
\hline
\end{tabular}

Table 2: AMZN : Estimated EMM parameters and respective estimation errors

\begin{tabular}{|c|c|c|c|c|c|c|c|}
\hline \multirow{2}{*}{$\begin{array}{l}\text { Date } \\
08-J U N-2005\end{array}$} & \multirow{2}{*}{$\begin{array}{l}\text { Model } \\
\text { BPRE }\end{array}$} & \multicolumn{3}{|c|}{ Parameters } & \multirow{2}{*}{ AAE } & \multirow{2}{*}{ APE } & \multirow{2}{*}{ RMSE } \\
\hline & & $\mathrm{p}$ & $\mathrm{a}$ & $\lambda$ & & & \\
\hline & \multirow{3}{*}{ lognormal } & 0.9786 & 0.9787 & 1.6634 & 0.0814 & 0.0325 & 0.0872 \\
\hline & & $\sigma$ & & & & & \\
\hline & & 0.2635 & & & 0.0845 & 0.0338 & 0.0903 \\
\hline \multirow[t]{4}{*}{ 13-JUL-2005 } & \multirow{4}{*}{$\begin{array}{l}\text { BPRE } \\
\text { lognormal }\end{array}$} & $\mathrm{p}$ & $\mathrm{a}$ & $\lambda$ & & & \\
\hline & & 0.9650 & 0.9651 & 1.9035 & 0.1195 & 0.0281 & 0.1269 \\
\hline & & $\sigma$ & & & & & \\
\hline & & 0.3445 & & & 0.1441 & 0.0340 & 0.1514 \\
\hline \multirow[t]{4}{*}{ 10-AUG-2005 } & \multirow{4}{*}{ lognormal } & $\mathrm{p}$ & a & $\lambda$ & & & \\
\hline & & 0.9143 & 0.9145 & 0.6477 & 0.0435 & 0.0131 & 0.0540 \\
\hline & & $\sigma$ & & & & & \\
\hline & & 0.2925 & & & 0.0541 & 0.0162 & 0.0675 \\
\hline \multirow[t]{4}{*}{ 14-SEP-2005 } & BPRE & $\mathrm{p}$ & $\mathrm{a}$ & $\lambda$ & & & \\
\hline & \multirow{3}{*}{ lognormal } & 0.9663 & 0.9664 & 1.8833 & 0.0391 & 0.0115 & 0.0481 \\
\hline & & $\sigma$ & & & & & \\
\hline & & 0.3177 & & & 0.0463 & 0.0137 & 0.0585 \\
\hline \multirow[t]{4}{*}{ 12-OCT-2005 } & BPRE & $\mathrm{p}$ & $\mathrm{a}$ & $\lambda$ & & & \\
\hline & \multirow{3}{*}{ lognormal } & 0.9387 & 0.9388 & 2.3054 & 0.0349 & 0.0074 & 0.0415 \\
\hline & & $\sigma$ & & & & & \\
\hline & & 0.4747 & & & 0.0771 & 0.0164 & 0.0885 \\
\hline
\end{tabular}


Table 3: INTC : Estimated EMM parameters and respective estimation errors

\begin{tabular}{llcccccc}
\hline Date & Model & \multicolumn{2}{c}{ Parameters } & & AAE & APE & RMSE \\
\hline 08-JUN-2005 & BPRE & $\mathrm{p}$ & $\mathrm{a}$ & $\lambda$ & & & \\
& & 0.9850 & 0.9851 & 1.5135 & 0.0329 & 0.0273 & 0.0361 \\
& lognormal & $\sigma$ \\
& & & & & & \\
& 0.2456 & & & 0.0219 & 0.0153 & 0.0274 \\
\hline 13-JUL-2005 & BPRE & $\mathrm{p}$ & $\mathrm{a}$ & $\lambda$ & & & \\
& & 0.9860 & 0.9861 & 1.4860 & 0.0452 & 0.0320 & 0.0548 \\
& lognormal & $\sigma$ \\
& 0.2308 & & & & & \\
& & & & 0.0379 & 0.0269 & 0.0453 \\
\hline 10-AUG-2005 & BPRE & $\mathrm{p}$ & $\mathrm{a}$ & $\lambda$ & & & \\
& & 0.9891 & 0.9892 & 1.3954 & 0.0499 & 0.0364 & 0.0516 \\
& lognormal & $\sigma$ & & & & & \\
& & 0.1895 & & & 0.0557 & 0.0407 & 0.0579 \\
\hline 14-SEP-2005 & BPRE & $\mathrm{p}$ & $\mathrm{a}$ & $\lambda$ & & & \\
& & 0.9859 & 0.9860 & 1.4893 & 0.0471 & 0.0425 & 0.0552 \\
& lognormal & $\sigma$ & & & & & \\
& & 0.2535 & & & 0.0564 & 0.0512 & 0.0577 \\
\hline 12-OCT-2005 & BPRE & $\mathrm{p}$ & $\mathrm{a}$ & $\lambda$ & & & \\
& & 0.9813 & 0.9814 & 1.6031 & 0.0492 & 0.0344 & 0.0612 \\
& lognormal & $\sigma$ \\
& & & & & & \\
& & 0.3091 & & & 0.0702 & 0.0493 & 0.0759 \\
\hline
\end{tabular}

Table 4: MSFT : Estimated EMM parameters and respective estimation errors

\begin{tabular}{llcccccc}
\hline Date & Model & \multicolumn{2}{c}{ Parameters } & & AAE & APE & RMSE \\
\hline 08-JUN-2005 & BPRE & $\mathrm{p}$ & $\mathrm{a}$ & $\lambda$ & & & \\
& & 0.9227 & 0.9235 & 0.1413 & 0.0670 & 0.0489 & 0.0791 \\
& lognormal & $\sigma$ \\
& & & & & & \\
& 0.1697 & & & 0.0748 & 0.0550 & 0.0862 \\
\hline 13-JUL-2005 & BPRE & $\mathrm{p}$ & $\mathrm{a}$ & $\lambda$ & & & \\
& & 0.9874 & 0.9875 & 1.2226 & 0.0350 & 0.0289 & 0.0355 \\
& lognormal & $\sigma$ \\
& 0.2009 & & & 0.0446 & 0.0371 & 0.0463 \\
\hline 10-AUG-2005 & BPRE & $\mathrm{p}$ & $\mathrm{a}$ & $\lambda$ & & & \\
& & 0.9375 & 0.9382 & 0.1898 & 0.0384 & 0.1568 & 0.0424 \\
& lognormal & $\sigma$ & & & & & \\
& & 0.1819 & & & 0.0591 & 0.0606 & 0.0730 \\
\hline 14-SEP-2005 & BPRE & $\mathrm{p}$ & $\mathrm{a}$ & $\lambda$ & & & \\
& & 0.9913 & 0.9915 & 1.3185 & 0.0212 & 0.0216 & 0.0213 \\
& lognormal & $\sigma$ & & & & & \\
& & 0.1740 & & & 0.0143 & 0.0146 & 0.0144 \\
\hline 12-OCT-2005 & BPRE & $\mathrm{p}$ & $\mathrm{a}$ & $\lambda$ & & & \\
& & 0.9103 & 0.9110 & 0.1945 & 0.0265 & 0.0278 & 0.0316 \\
& lognormal & $\sigma$ & & & & & \\
& & 0.2298 & & & 0.0409 & 0.0430 & 0.0506 \\
\hline
\end{tabular}


Table 5: SPX : Up-and-Out and Standard Call Option Prices

\begin{tabular}{|c|c|c|c|c|c|c|}
\hline \multicolumn{7}{|c|}{ U Up-and-Out Call Option Prices } \\
\hline \multirow[b]{2}{*}{ Strike } & \multicolumn{2}{|c|}{5 days to Maturity } & \multicolumn{2}{|c|}{38 days to Maturity } & \multicolumn{2}{|c|}{72 days to Maturity } \\
\hline & BPRE & lognormal & BPRE & lognormal & BPRE & lognormal \\
\hline 1140 & 87.8365 & 88.0095 & 73.9546 & 70.2746 & 53.3410 & 49.0356 \\
\hline 1160 & 67.8538 & 68.0250 & 56.9195 & 53.7793 & 40.3445 & 36.7079 \\
\hline 1180 & 47.8757 & 48.0417 & 40.6091 & 38.0147 & 28.3509 & 25.4005 \\
\hline 1200 & 28.0554 & 28.1845 & 25.9172 & 23.8965 & 17.9533 & 15.7139 \\
\hline 1220 & 10.4329 & 10.6313 & 14.0213 & 12.5981 & 9.7494 & 8.2266 \\
\hline 1240 & 1.6013 & 1.6579 & 5.8552 & 5.0065 & 4.1346 & 3.2765 \\
\hline 1260 & 0.0979 & 0.0719 & 1.5141 & 1.1501 & 1.0921 & 0.7582 \\
\hline 1270 & 0.0018 & 0.0005 & 0.0962 & 0.0444 & 0.0706 & 0.0295 \\
\hline \multicolumn{7}{|c|}{ Standard Call Option Prices } \\
\hline & \multicolumn{2}{|c|}{5 days to Maturity } & \multicolumn{2}{|c|}{ (38 days to Maturity } & \multicolumn{2}{|c|}{ "72 days to Maturity } \\
\hline Strike & BPRE & lognormal & BPRE & lognormal & BPRE & lognormal \\
\hline 1140 & 88.0133 & 88.0133 & 93.7358 & 93.7159 & 100.0277 & 99.9622 \\
\hline 1160 & 68.0284 & 68.0283 & 74.1869 & 74.1452 & 81.2017 & 81.1114 \\
\hline 1180 & 48.0482 & 48.0445 & 55.3628 & 55.3053 & 63.3820 & 63.2865 \\
\hline 1200 & 28.2256 & 28.1868 & 38.1574 & 38.1121 & 47.1707 & 47.1026 \\
\hline 1220 & 10.6010 & 10.6330 & 23.7500 & 23.7426 & 33.1932 & 33.1817 \\
\hline 1240 & 1.7564 & 1.6591 & 13.0843 & 13.1021 & 21.9192 & 21.9705 \\
\hline 1260 & 0.2344 & 0.0727 & 6.3007 & 6.2990 & 13.5034 & 13.5943 \\
\hline 1270 & 0.1196 & 0.0007 & 2.6617 & 2.6066 & 7.7357 & 7.8258 \\
\hline
\end{tabular}

The current value $S(0)$ of the SPX is 1227.2 and the risk-free rate $r$ is 0.0377 per annum. The barrier level is 1290 in order to be greater than all of the selected strike prices. 
Table 6: AMZN : Up-and-Out and Standard Call Option Prices

\begin{tabular}{|c|c|c|c|c|c|c|}
\hline \multicolumn{7}{|c|}{ Up-and-Out Call Option Prices } \\
\hline \multirow[b]{2}{*}{ Strike } & \multicolumn{2}{|c|}{5 days to Maturity } & \multicolumn{2}{|c|}{38 days to Maturity } & \multicolumn{2}{|c|}{72 days to Maturity } \\
\hline & BPRE & lognormal & BPRE & lognormal & BPRE & lognormal \\
\hline 39 & 4.0173 & 4.0962 & 2.3249 & 1.9097 & 1.3452 & 1.0368 \\
\hline 40 & 3.0510 & 3.1285 & 1.8005 & 1.4481 & 1.0270 & 0.7718 \\
\hline 41 & 2.1410 & 2.2189 & 1.3410 & 1.0517 & 0.7551 & 0.5511 \\
\hline 42 & 1.3476 & 1.4268 & 0.9519 & 0.7240 & 0.5301 & 0.3738 \\
\hline 43 & 0.7394 & 0.8116 & 0.6360 & 0.4656 & 0.3507 & 0.2373 \\
\hline 44 & 0.3475 & 0.3989 & 0.3923 & 0.2735 & 0.2146 & 0.1379 \\
\hline 45 & 0.1388 & 0.1649 & 0.2167 & 0.1412 & 0.1178 & 0.0706 \\
\hline 46 & 0.0462 & 0.0548 & 0.1013 & 0.0597 & 0.0548 & 0.0297 \\
\hline \multicolumn{7}{|c|}{ Standard Call Option Prices } \\
\hline & \multicolumn{2}{|c|}{5 days to Maturity } & \multicolumn{2}{|c|}{38 days to Maturity } & \multicolumn{2}{|c|}{72 days to Maturity } \\
\hline Strike & BPRE & lognormal & BPRE & lognormal & BPRE & lognormal \\
\hline 39 & 4.1412 & 4.1369 & 4.8860 & 4.8689 & 5.6102 & 5.5882 \\
\hline 40 & 3.1726 & 3.1652 & 4.1298 & 4.1182 & 4.9179 & 4.9069 \\
\hline 41 & 2.2603 & 2.2515 & 3.4395 & 3.4348 & 4.2772 & 4.2789 \\
\hline 42 & 1.4646 & 1.4553 & 2.8213 & 2.8239 & 3.6905 & 3.7053 \\
\hline 43 & 0.8541 & 0.8361 & 2.2790 & 2.2879 & 3.1589 & 3.1865 \\
\hline 44 & 0.4599 & 0.4193 & 1.8132 & 1.8264 & 2.6825 & 2.7217 \\
\hline 45 & 0.2488 & 0.1813 & 1.4221 & 1.4367 & 2.2603 & 2.3091 \\
\hline 46 & 0.1539 & 0.0671 & 1.1010 & 1.1136 & 1.8904 & 1.9462 \\
\hline
\end{tabular}

The current price $S(0)$ of the AMZN is 43.10 and the risk-free rate $r$ is 0.0377 per annum. The barrier level is 49 in order to be greater than all of the selected strike prices. 
Table 7: INTC : Up-and-Out and Standard Call Option Prices

\begin{tabular}{|c|c|c|c|c|c|c|}
\hline \multicolumn{7}{|c|}{ "Up-and-Out Call Option Prices } \\
\hline \multirow[b]{2}{*}{ Strike } & \multicolumn{2}{|c|}{5 days to Maturity } & \multicolumn{2}{|c|}{38 days to Maturity } & \multicolumn{2}{|c|}{72 days to Maturity } \\
\hline & BPRE & lognormal & BPRE & lognormal & BPRE & lognormal \\
\hline 23.00 & 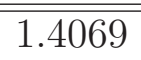 & 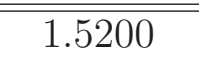 & 1.7159 & 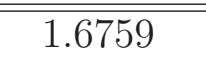 & 1.6180 & 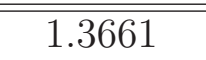 \\
\hline 23.50 & 0.9455 & 1.0580 & 1.3699 & 1.3459 & 1.3267 & 1.1102 \\
\hline 24.00 & 0.5490 & 0.6570 & 1.0652 & 1.0548 & 1.0662 & 0.8836 \\
\hline 24.50 & 0.2641 & 0.3530 & 0.8047 & 0.8046 & 0.8378 & 0.6867 \\
\hline 25.00 & 0.1045 & 0.1598 & 0.5889 & 0.5953 & 0.6414 & 0.5190 \\
\hline 25.50 & 0.0346 & 0.0599 & 0.4160 & 0.4254 & 0.4765 & 0.3797 \\
\hline 26.00 & 0.0099 & 0.0184 & 0.2823 & 0.2918 & 0.3415 & 0.2670 \\
\hline 26.50 & 0.0025 & 0.0046 & 0.1829 & 0.1905 & 0.2342 & 0.1786 \\
\hline \multicolumn{7}{|c|}{ "Standard Call Option Prices } \\
\hline & \multicolumn{2}{|c|}{ (5 days to Maturity } & \multicolumn{2}{|c|}{38 days to Maturity } & \multicolumn{2}{|c|}{72 days to Maturity } \\
\hline Strike & BPRE & lognormal & BPRE & lognormal & BPRE & lognormal \\
\hline 23.00 & 1.5219 & 1.5200 & 1.9352 & 1.9557 & 2.2888 & 2.3274 \\
\hline 23.50 & 1.0605 & 1.0580 & 1.5820 & 1.6058 & 1.9597 & 2.0041 \\
\hline 24.00 & 0.6640 & 0.6570 & 1.2702 & 1.2948 & 1.6615 & 1.7102 \\
\hline 24.50 & 0.3790 & 0.3530 & 1.0026 & 1.0247 & 1.3953 & 1.4463 \\
\hline 25.00 & 0.2191 & 0.1598 & 0.7796 & 0.7956 & 1.1613 & 1.2119 \\
\hline 25.50 & 0.1490 & 0.0599 & 0.5995 & 0.6059 & 0.9588 & 1.0064 \\
\hline 26.00 & 0.1239 & 0.0184 & 0.4587 & 0.4525 & 0.7864 & 0.8283 \\
\hline 26.50 & 0.1163 & 0.0046 & 0.3522 & 0.3315 & 0.6420 & 0.6756 \\
\hline
\end{tabular}

The current price $S(0)$ of the INTC is 24.49 and the risk-free rate $r$ is 0.0377 per annum. The barrier level is 30 in order to be greater than all of the selected strike prices. 
Table 8: MSFT : Up-and-Out and Standard Call Option Prices

\begin{tabular}{|c|c|c|c|c|c|c|}
\hline \multicolumn{7}{|c|}{ "Up-and-Out Call Option Prices } \\
\hline \multirow[b]{2}{*}{ Strike } & \multicolumn{2}{|c|}{5 days to Maturity } & \multicolumn{2}{|c|}{38 days to Maturity } & \multicolumn{2}{|c|}{72 days to Maturity } \\
\hline & BPRE & lognormal & BPRE & lognormal & BPRE & lognormal \\
\hline 25.00 & 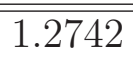 & 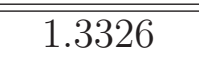 & 1.5696 & 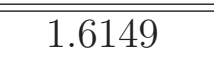 & 1.7205 & 1.6518 \\
\hline 25.50 & 0.8000 & 0.8576 & 1.1982 & 1.2469 & 1.3829 & 1.3280 \\
\hline 26.00 & 0.3975 & 0.4534 & 0.8787 & 0.9292 & 1.0844 & 1.0425 \\
\hline 26.50 & 0.1427 & 0.1819 & 0.6166 & 0.6661 & 0.8275 & 0.7968 \\
\hline 27.00 & 0.0374 & 0.0518 & 0.4125 & 0.4579 & 0.6126 & 0.5910 \\
\hline 27.50 & 0.0076 & 0.0100 & 0.2624 & 0.3010 & 0.4384 & 0.4235 \\
\hline 28.00 & 0.0012 & 0.0013 & 0.1582 & 0.1883 & 0.3017 & 0.2915 \\
\hline 28.50 & 0.0002 & 0.0001 & 0.0900 & 0.1115 & 0.1983 & 0.1909 \\
\hline \multicolumn{7}{|c|}{ Standard Call Option Prices } \\
\hline & \multicolumn{2}{|c|}{5 days to Maturity } & \multicolumn{2}{|c|}{38 days to Maturity } & \multicolumn{2}{|c|}{7272 days to Maturity } \\
\hline Strike & BPRE & lognormal & BPRE & lognormal & BPRE & lognormal \\
\hline 25.00 & 1.3342 & 1.3326 & 1.6395 & 1.6452 & 1.9252 & 1.9374 \\
\hline 25.50 & 0.8600 & 0.8576 & 1.2674 & 1.2752 & 1.5777 & 1.5935 \\
\hline 26.00 & 0.4575 & 0.4534 & 0.9472 & 0.9552 & 1.2693 & 1.2878 \\
\hline 26.50 & 0.2024 & 0.1819 & 0.6844 & 0.6900 & 1.0025 & 1.0220 \\
\hline 27.00 & 0.0964 & 0.0518 & 0.4797 & 0.4797 & 0.7777 & 0.7960 \\
\hline 27.50 & 0.0658 & 0.0100 & 0.3289 & 0.3205 & 0.5935 & 0.6084 \\
\hline 28.00 & 0.0588 & 0.0013 & 0.2240 & 0.2057 & 0.4470 & 0.4562 \\
\hline 28.50 & 0.0571 & 0.0001 & 0.1551 & 0.1268 & 0.3337 & 0.3357 \\
\hline
\end{tabular}

The current price $S(0)$ of the MSFT is 26.31 and the risk-free rate $r$ is 0.0377 per annum. The barrier level is 32 in order to be greater than all of the selected strike prices. 


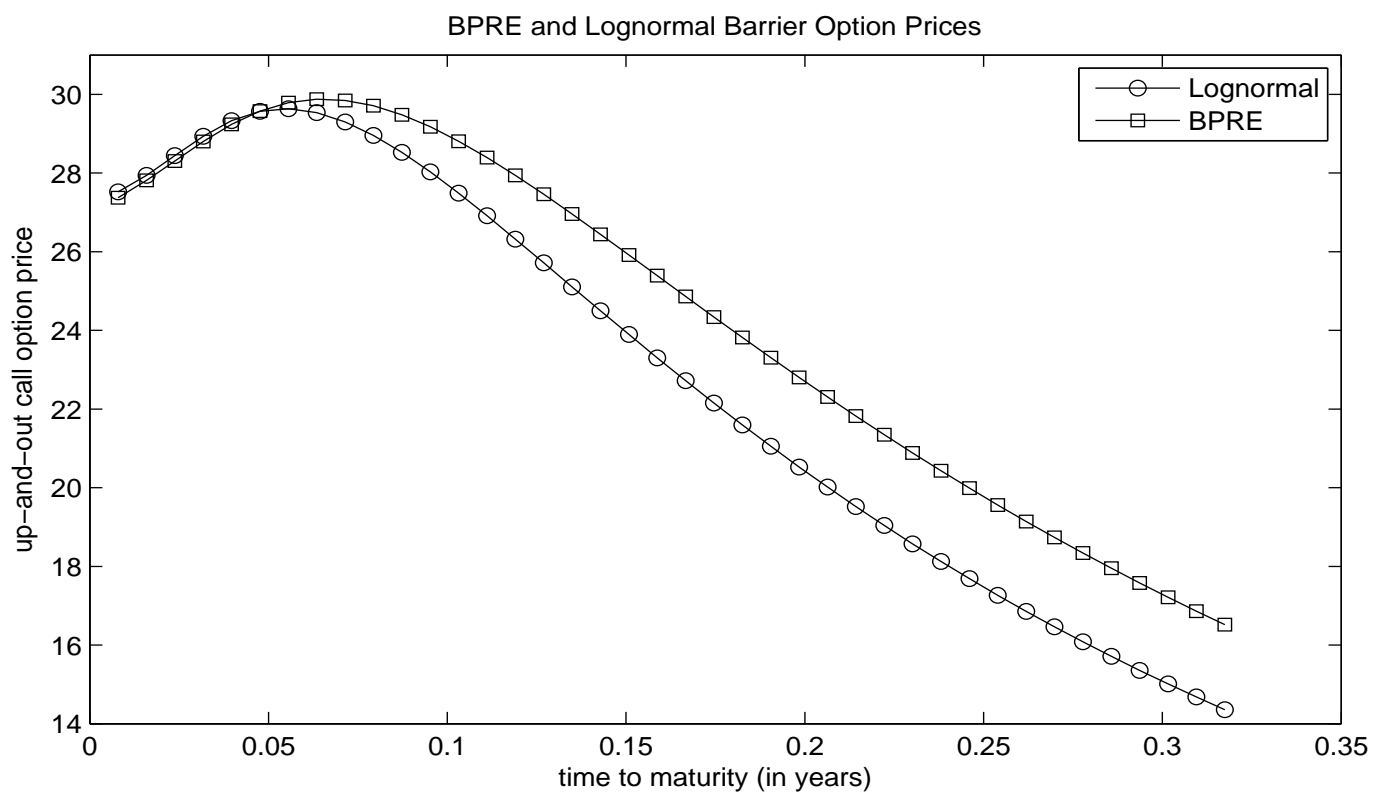

Figure 1: Up-and-out call option on SPX prices from BPRE model and lognormal model as a function of time to maturity. The current value $S(0)$ of the SPX is 1227.2, the risk-free rate $r$ is 0.0377 per annum, the strike price is 1200, and the barrier level is 1290 .

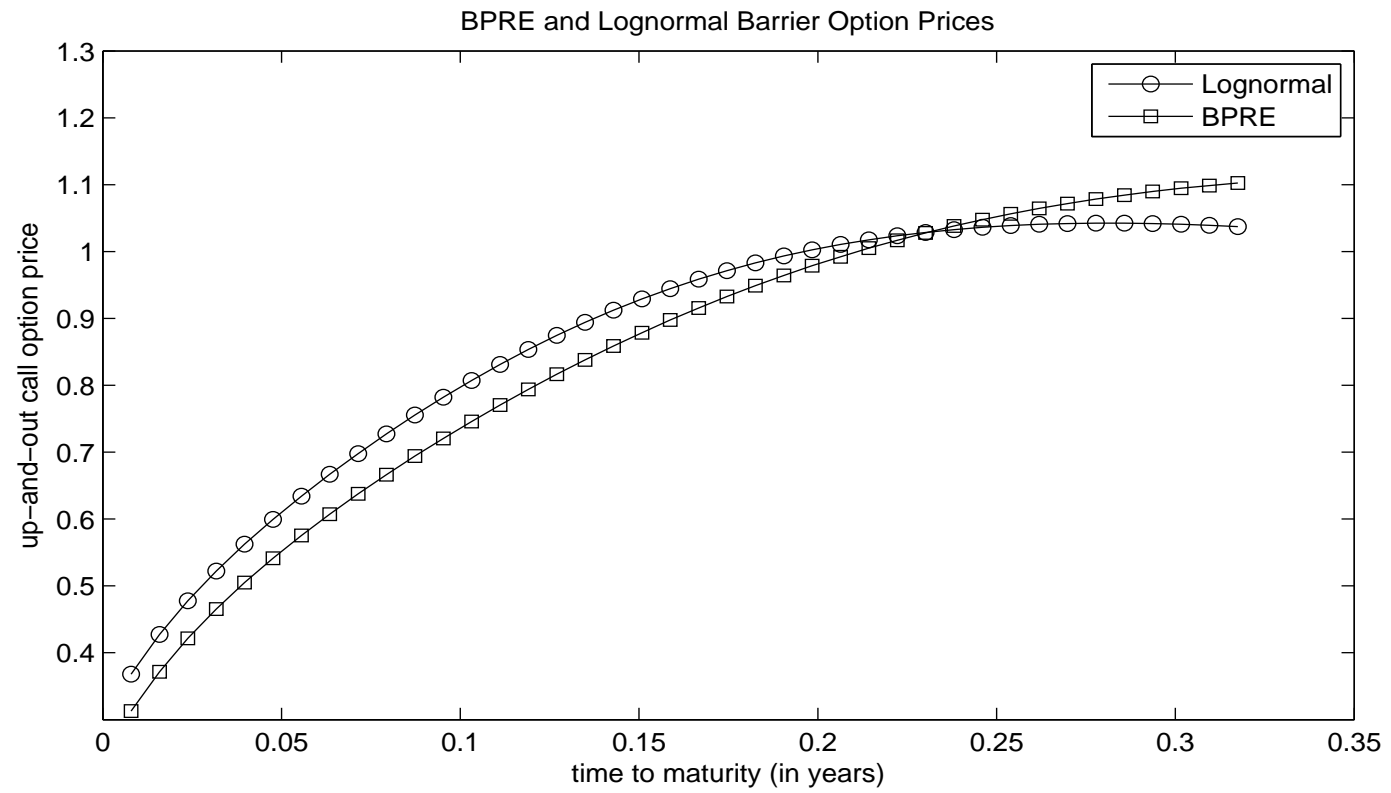

Figure 2: Up-and-out call option on AMZN prices from BPRE model and lognormal model as a function of time to maturity. The current price $S(0)$ of the AMZN is 43.1, the risk-free rate $r$ is 0.0377 per annum, the strike price is 42 , and the barrier level is 49 . 


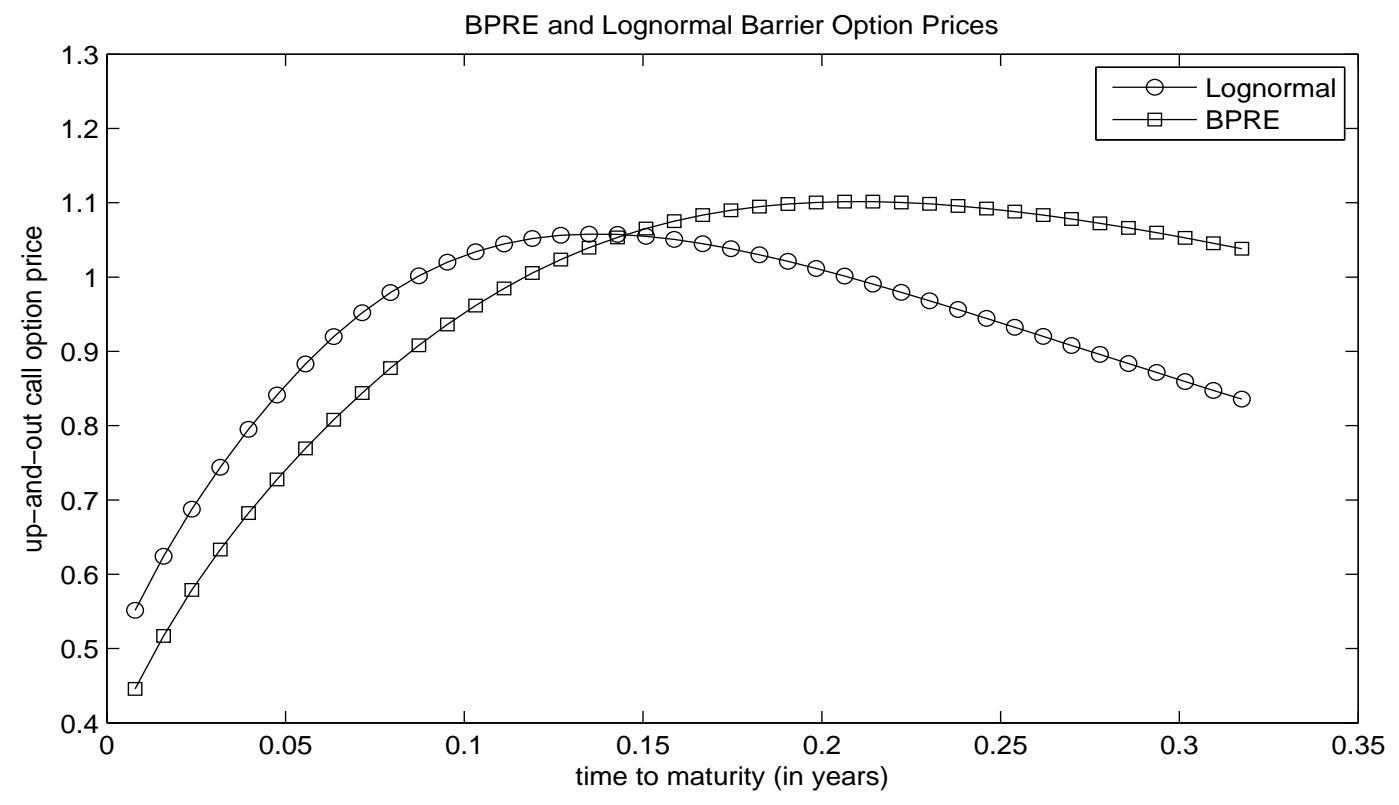

Figure 3: Up-and-out call option on INTC prices from BPRE model and lognormal model as a function of time to maturity. The current price $S(0)$ of the INTC is 24.49, the risk-free rate $r$ is 0.0377 per annum, the strike price is 24 , and the barrier level is 30 .

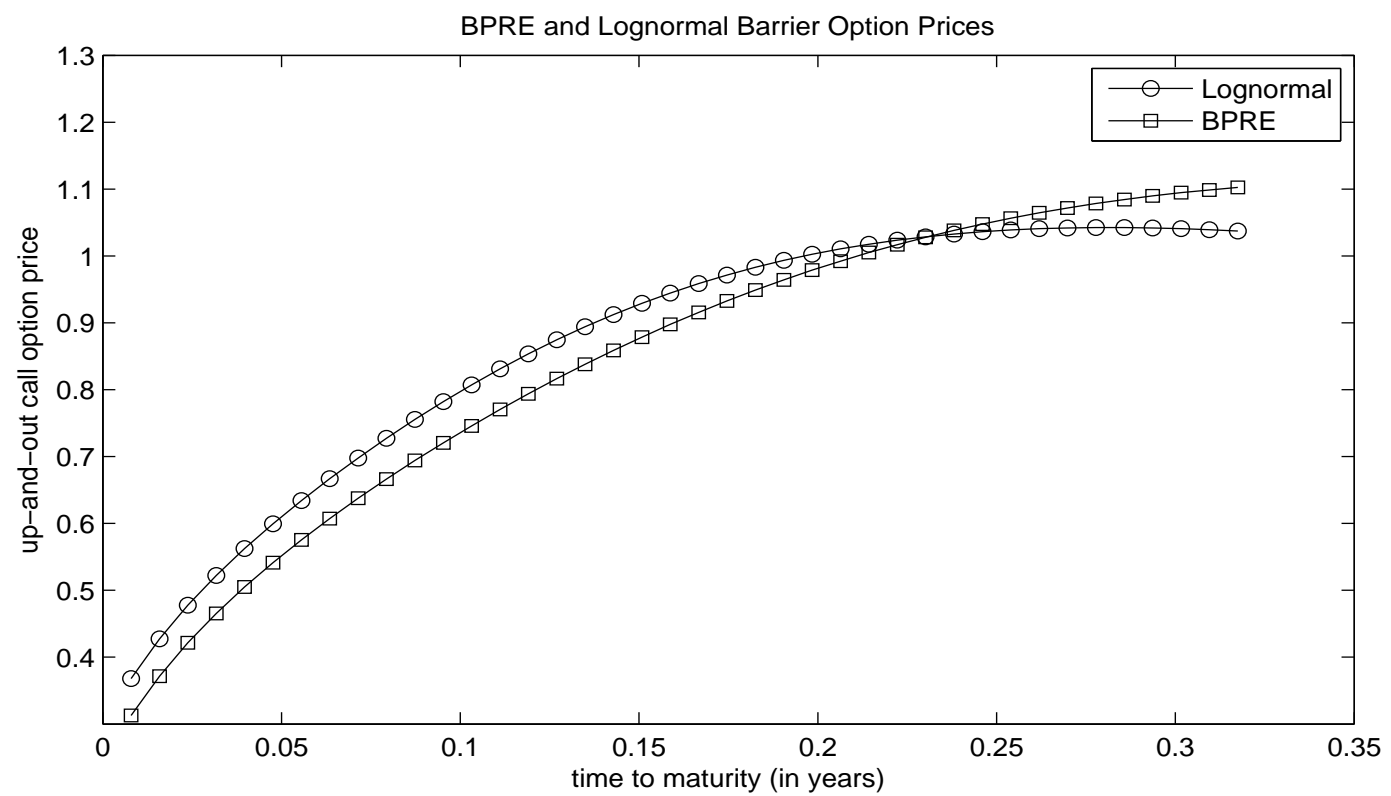

Figure 4: Up-and-out call option on MSFT prices from BPRE model and lognormal model as a functio,n of time to maturity. The current price $S(0)$ of the MSFT is 26.31, the risk-free rate $r$ is 0.0377 per annum, the strike price is 26 , and the barrier level is 32 . 